\title{
Extensivity in infinitely large multiplex networks
}

Maria Angélica Araujo* (1) and Murilo S. Baptista

${ }^{*}$ Correspondence:

mariaaaraujo13@gmail.com Institute of Complex Sciences and Mathematical Biology, University of Aberdeen, SUPA, AB24 3UE Aberdeen, UK

\begin{abstract}
In this paper, we investigate the relationship between the coupling strengths and the extensive behaviour of the sum of the positive Lyapunov exponents of multiplex networks formed by coupled dynamical units. Considering networks where the dynamics of the nodes is given by the shift map, we do not only demonstrate which are the relevant parameters leading to extensivity, but also provide exact formulas how they are related. A distinct result was to show that it is always possible to construct infinitely large extensive networks by attaching, with rescaled inter-connections, infinitely many smaller networks. These smaller networks are effectively the building blocks of the large network. This is because these building blocks can have arbitrary topology and the strength of connections among nodes only depends on the block size, and not on the size of the whole network.
\end{abstract}

Keywords: Extensivity, Multiplex networks, Lyapunov exponents

\section{Introduction}

Complex networks are structures consisting of nodes and edges, which are connected in a non-trivial way (Boccaletti et al. 2006; E J Newman 2010; Strogatz 2001; Estrada 2015). The theory of complex networks has been studied across many fields, such as sociology, biology, mathematics, physics and computer science and a wide variety of phenomena are described by complex networks, from a microscopic level as in complex brain networks (Bullmore and Sporns 2009) to macroscopic systems of social interactions (Robins et al. 2007) and technological systems (Nardelli et al. 2014), for instance.

An important concept that can play a significant role in the understanding of complex dynamical networks is extensivity. A quantity is extensive, if it scales directly with the size of the system. Extensive quantities are additive for subsystems, which means that, physical systems that are extensive can be decomposed into independent subsystems that could also be extensive. The reverse is also true. Extensive systems can be constructed by putting together independent subsystems, for example, adding more mass to a body.

The concept of extensive quantities can be extended to the scope of complex systems. Ruelle was the first to infer the extensivity of chaos (Ruelle 1982). The most common and practical procedure to identify chaos in complex systems is computing the Lyapunov Exponents (LEs), which measure the rate of separation of infinitesimal close trajectories in phase space (Eckmann and Ruelle 1985; Ott 2002). Then, according to Ruelle, extensivity can be understood by studying the curve of LEs, when arranged in descending order,

(c) The Author(s). 2019 Open Access This article is distributed under the terms of the Creative Commons Attribution 4.0 International License (http://creativecommons.org/licenses/by/4.0/), which permits unrestricted use, distribution, and reproduction in any medium, provided you give appropriate credit to the original author(s) and the source, provide a link to the Creative Commons license, and indicate if changes were made. 
as function of the their normalised index and calculated at different system sizes. If these curves collapse onto a single asymptotic spectrum as the system size grows, then chaos is considered to be extensive. A direct consequence of this is that, the sum of the positive LEs is linearly related to the size of the network. Moreover, extensive systems can be typically decomposed into smaller fundamental units that could imply that a large dynamical network with complex topology could be broken down into "independent" subnetworks with similar dynamic properties.

Extensive chaos has been discussed in many complex systems. In globally coupled dynamical systems, although all the elements of the system are subject to the same influences, the nontrivial connection between their components can give rise to collective chaos (Shibata and Kaneko 1998) and nonextensive behaviour (Takeuchi et al. 2011). Due to the nontrivial collective behaviour in these type of systems, the extensivity of the Lyapunov exponents has been contested in (Takeuchi et al. 2009). In contrast to this, extensivity has been commonly observed, for instance, by studying the spatiotemporal chaos, in Rayleigh-Bénard convection (Paul et al. 2007) and in reaction-diffusion networks (Stahlke and Wackerbauer 2009; 2011).

For high-dimension systems, we can mention the results in (Karimi and Paul 2010), where the authors investigated the extensive chaos behaviour of the Lorenz-96 model by studying the variation of the fractal dimension for different parameters of the system. In (Xi et al. 2000), Xi et al. studied the extensivity of the Lyapunov dimension and the Kolmogorov-Sinai entropy in the one-dimensional Nikolaevskii model. Extensivity of chaos was also detected in large sparse neuron networks (Monteforte and Wolf 2010) and in different classes of sparse random networks (Luccioli et al. 2012). In the latter, the authors have explored the relationship between the number of incoming connections per node and the extensive behaviour, when all nodes are subject to the same connectivity.

In this paper, we study analytically how extensivity of the sum of the positive Lyapunov exponents of networks of coupled shift maps, but where the topology of the couplings follows a "multiplex" topology, can be maintained by smartly changing the configuration of the network as it grows in size. So, the topology of the constructed network of interacting units is a network of many subnetworks. A subnetwork is a set of dynamical units coupled by an intra topology and an intra coupling strength. Subnetworks are connected by the so called inter topology with inter coupling strength. A main point of interest in this work is to understand the role of the intra and inter-couplings in the extensive behaviour of large multiplex networks. Our extensive quantity, the sum of the positive Lyapunov exponents, denoted by $H_{K S}$, is an upper bound for the Kolmogorov-Sinai entropy. In contrast to the entropy, $H_{K S}$ can be usually well estimated, even in networks with arbitrary sizes.

At variance with Antonopoulos and Baptista (2017), which have explored this relationship in multiplex networks formed only by two layers of nodes, we analyze in this work infinitely large multiplex networks. We start with an initial network formed by two layers, as described in (Antonopoulos and Baptista 2017), and then, in each step, we duplicate the network that we had before, and connect the two subnetworks, maintaining the same intra and inter structure. We show that extensivity only depends on the coupling strengths, and that other quantities which proved to be important to maintain extensivity in (Antonopoulos and Baptista 2017), such as the sum of the intra and inter-degrees of the nodes of the layers, do not interfere in this behaviour. 
Moreover, we also show that, given an initial dynamical network with an arbitrary intra-connectivity topology, it is always possible to construct an infinitely large extensive network with an infinite number of these initial networks, the building blocks, connected with rescaled inter-link strengths. This result reinforces the idea that an extensive complex network can be broken down into building blocks that are also extensive. Since all our calculations are exact, we provide a reliable and practical method to achieve extensivity.

\section{Methods}

Consider, initially, a network $\Omega^{(0)}$ with $N_{0}$ nodes where the dynamics of the nodes is given by the shift $\operatorname{map} F\left(x_{n}^{(i)}\right)=2 x_{n}^{(i)}(\bmod 1)$, i.e.:

$$
x_{n+1}^{(i)}=2 x_{n}^{(i)}-\varepsilon \sum_{j=1}^{N_{0}} A_{i j} x_{n}^{(j)}(\bmod 1),
$$

where $\varepsilon$ represents the coupling strength of the intra-connections and $\mathbf{A}=\left(A_{i j}\right)$ denotes the Laplacian matrix. The main motivation to use this map is the fact that it is a transformation with constant Jacobian, allowing for an analytical calculation of the LEs for networks constructed with this map. Moreover, this paper extends previous results in the works (Antonopoulos and Baptista 2017) and (Baptista et al. 2016). The $(\bmod 1)$ is a modular function that guarantees that the transformed point (by $2 x$ ) remains in the interval $[0,1]$.

Now, let $\Omega^{(1)}$ be the network constructed by coupling two equal subnetworks $\Omega^{(0)}$, then its number of nodes is $N_{1}=2 N_{0}$ and it can be represented by:

$$
x_{n+1}^{(i)}=2 x_{n}^{(i)}-\varepsilon \sum_{j=1}^{N_{1}} G_{i j} x_{n}^{(j)}-\gamma \alpha \sum_{j=1}^{N_{1}} L_{i j} x_{n}^{(j)}(\bmod 1),
$$

where $\gamma$ is the coupling strength of the inter-connections, $\alpha=\frac{l_{12}}{N_{0}}$ is the ratio between the number of inter-connections $l_{12}$ and $N_{0}$. The Laplacian matrices of the intra and interconnections are represented by $\mathbf{G}=\left(G_{i j}\right)$ and $\mathbf{L}=\left(L_{i j}\right)$, respectively. Therefore, they are given by:

$$
\mathbf{G}=\left(\begin{array}{ll}
\mathbf{A} & \mathbf{0} \\
\mathbf{0} & \mathbf{A}
\end{array}\right) \text { and } \mathbf{L}=\left(\begin{array}{ll}
\mathbf{D}_{\mathbf{1}} & -\mathbf{B} \\
-\mathbf{B}^{T} & \mathbf{D}_{\mathbf{2}}
\end{array}\right)
$$

where $\mathbf{0}$ denotes the $N_{0} \times N_{0}$ zero matrix, $T$ stands for the transpose and $\mathbf{B}$ represents the adjacency matrix of the inter-connections. The matrices $\mathbf{D}_{\mathbf{1}}$ and $\mathbf{D}_{2}$ are the diagonal degree matrices of the adjacency matrices $\mathbf{B}$ and $\mathbf{B}^{T}$, respectively. Their components are defined as:

$$
\left(D_{1}\right)_{i i}=\sum_{j=1}^{N_{0}} B_{i j} \quad \text { and } \quad\left(D_{2}\right)_{i i}=\sum_{j=1}^{N_{0}} B_{i j}^{T}, \quad \text { for } \quad i=1, \ldots, N_{0} .
$$

We suppose that the intra and inter-connections are undirected, i.e., each connection between the nodes is bidirectional. Besides, we consider a diagonal interlinking configuration, which means that each node in a subnetwork is only connected to the corresponding node in the other equal subnetwork, consequently $l_{12}=N_{0}$ and $\alpha=1$. Figure 1 illustrates this configuration in networks where the building blocks have $N_{0}=6$ nodes with ring topology in (a) and all-to-all topology in (b). 


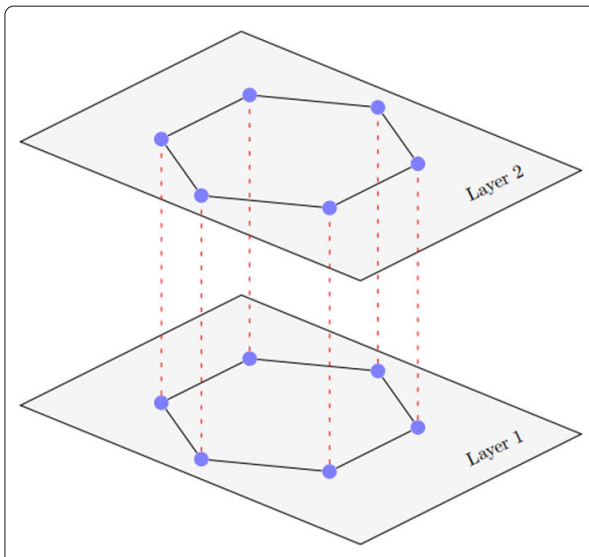

a

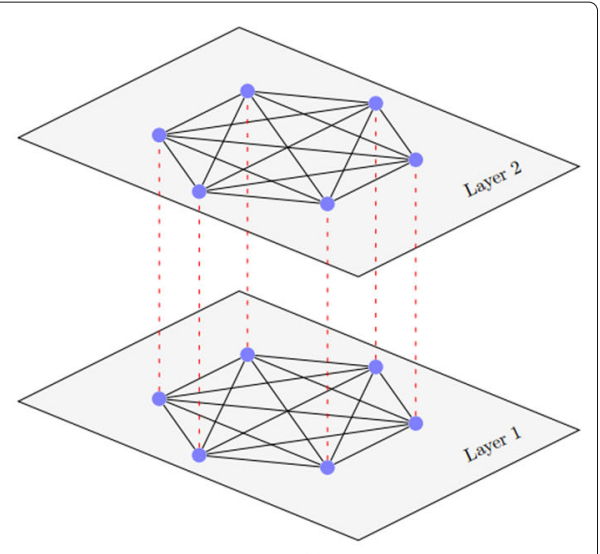

b

Fig. 1 Examples of the diagonal interlink configuration. Each network is formed by two equal subnetworks with $N_{0}=6$ nodes each and, with (a) ring and (b) all-to-all topology. The solid black and dashed red links correspond to the intra and inter-connections, respectively

We can rewrite Eq. (2) in a matrix form as:

$$
\mathbf{x}_{n+1}=2 \mathbf{x}_{n}-\mathbf{M}_{1} \mathbf{x}_{n}(\bmod 1),
$$

where $\mathbf{M}_{1}=\left[\begin{array}{cc}\varepsilon \mathbf{A}+\gamma \alpha \mathbf{D}_{\mathbf{1}} & -\gamma \alpha \mathbf{B} \\ -\gamma \alpha \mathbf{B}^{T} & \varepsilon \mathbf{A}+\gamma \alpha \mathbf{D}_{\mathbf{2}}\end{array}\right]$. Defining $\mathbf{J}_{1}=2 \mathbf{I}_{1}-\mathbf{M}_{1}$, where $\mathbf{I}_{1}$ represents the $N_{1} \times N_{1}$ identity matrix, Eq. (5) becomes:

$$
\mathbf{x}_{n+1}=\mathbf{J}_{1} \mathbf{x}_{n}(\bmod 1) .
$$

Since all the connections are undirected, the matrix $\mathbf{M}_{1}$ is symmetric as well as the Jacobian matrix $\mathbf{J}_{1}$. The Lyapunov exponent of Eq. (6) in the direction of a vector $\mathbf{v} \in \mathbb{R}^{N_{1}}$ is given by, (Ott 2002):

$$
\lambda(\mathbf{v})=\lim _{N \longrightarrow \infty} \frac{1}{N} \ln \left\|\mathbf{J}_{1}^{N} \cdot \mathbf{v}\right\|,
$$

where $\|\cdot\|$ represents the Euclidean norm in $\mathbb{R}^{N_{1}}$.

Let $\lambda_{i}^{(1)}$, for $i=0, \ldots, N_{1}-1$, be the unordered (and possibly not all distinct) Lyapunov exponents of Eq. (6). As consequence of the Jacobian $\mathbf{J}_{1}$ being constant and symmetric we have that:

$$
\lambda_{i}^{(1)}=\ln \left|\theta_{i}^{(1)}\right|, \quad \text { for } i=0, \ldots, N_{1}-1,
$$

where $\theta_{i}^{(1)}$ represents the eigenvalues of the matrix $\mathbf{J}_{1}$ and $|\cdot|$ denotes the absolute value. This result is demonstrated in (Araujo and Baptista: A comprehensive review about Lyapunov Exponents in continuous and discrete dynamical networks, in preparation). Note that, if we denote by $\mu_{i}^{(1)}$ the eigenvalues of $\mathbf{M}_{1}$ for $i=0, \ldots, N_{1}-1$, then:

$$
\theta_{i}^{(1)}=2-\mu_{i}^{(1)} \text {. }
$$

Thus, Eq. (8) can be written as:

$$
\lambda_{i}^{(1)}=\ln \left|2-\mu_{i}^{(1)}\right| .
$$

By (Baptista et al. 2016; Martín-Hernández et al. 2014), the unordered eigenvalues of $\mathbf{M}_{1}$ are given by: 


$$
\begin{aligned}
\mu_{2 i}^{(1)} & =\varepsilon \omega_{i} \\
\mu_{2 i+1}^{(1)} & =\varepsilon \omega_{i}+2 \gamma \alpha,
\end{aligned}
$$

for $i=0, \ldots, N_{0}-1$ and where $0=\omega_{0} \leqslant \omega_{1} \leqslant \ldots \leqslant \omega_{N_{0}-1}$ represent the ordered eigenvalues of the Laplacian matrix A. Note that, $\mu_{2 i}^{(1)}$ are the eigenvalues coming from one layer, whereas $\mu_{2 i+1}^{(1)}$ are the eigenvalues due to the inter-connectivity, generated by perturbing $\mu_{2 i}^{(1)}$ with the term $2 \gamma \alpha$.

The main goal of this work is to grow the network $\Omega^{(1)}$ and to study the conditions to have the sum of the positive LEs of each network linearly proportional to its number of nodes. This growing is made as following: $\Omega^{(2)}$ is constructed by coupling two equal subnetworks $\Omega^{(1)}$ with the same parameter $\alpha$ and with the same diagonal interlinking configuration. The number of nodes of $\Omega^{(2)}$ is $N_{2}=2 N_{1}=2^{2} N_{0}$ and it can be represented by:

$$
\mathbf{x}_{n+1}=2 \mathbf{x}_{n}-\mathbf{M}_{2} \mathbf{x}_{n} \equiv \mathbf{J}_{2} \mathbf{x}_{n}(\bmod 1),
$$

where the matrices $\mathbf{M}_{2}$ and $\mathbf{J}_{2}$ are constructed in the same way as $\mathbf{M}_{1}$ and $\mathbf{J}_{1}$, respectively. The eigenvalues of $\mathbf{M}_{2}$ are given by:

$$
\begin{aligned}
\mu_{4 i}^{(2)} & =\varepsilon \omega_{i} \\
\mu_{4 i+1}^{(2)} & =\varepsilon \omega_{i}+2 \gamma \alpha \\
\mu_{4 i+2}^{(2)} & =\varepsilon \omega_{i}+2 \gamma \alpha \\
\mu_{4 i+3}^{(2)} & =\varepsilon \omega_{i}+4 \gamma \alpha,
\end{aligned}
$$

for $i=0, \ldots, N_{0}-1$. Similar to how the eigenvalues of $\mathbf{M}_{1}$ were generated, the set $\mu^{(2)} \equiv$ $\left\{\mu_{4 i}^{(2)}, \mu_{4 i+1}^{(2)}\right\}$ are the eigenvalues due to the layer $\Omega^{(1)}$ and $\left\{\mu_{4 i+2}^{(2)}, \mu_{4 i+3}^{(2)}\right\}$ are generated by perturbing the set $\mu^{(2)}$ with $2 \gamma \alpha$.

Proceeding this way, the network $\Omega^{(k)}$ with a number of nodes given by $N_{k}=2^{k} N_{0}$ is constructed by coupling two equal subnetworks $\Omega^{(k-1)}$ with the same parameters and configuration. Thus, as a result of this recursive procedure for generating self-similar networks each layer is connected to all the other layers in the network, and Fig. 1 represents only the first step of this process. The inter-coupling strength, $\gamma$, will be rescaled in order to maintain the extensive property of the network as it grows. The distinct eigenvalues of the corresponding matrix $\mathbf{M}_{k}$ for the network $\Omega^{(k)}$ are:

$$
\begin{aligned}
\tilde{\mu}_{0, i}^{(k)} & \equiv \varepsilon \omega_{i} \\
\tilde{\mu}_{1, i}^{(k)} & \equiv \varepsilon \omega_{i}+2 \gamma \alpha \\
\tilde{\mu}_{2, i}^{(k)} & \equiv \varepsilon \omega_{i}+4 \gamma \alpha \\
& \vdots \\
\tilde{\mu}_{j, i}^{(k)} & \equiv \varepsilon \omega_{i}+2 j \gamma \alpha \\
& \vdots \\
\tilde{\mu}_{j=(k-1), i}^{(k)} & \equiv \varepsilon \omega_{i}+2(k-1) \gamma \alpha \\
\tilde{\mu}_{j=k, i}^{(k)} & \equiv \varepsilon \omega_{i}+2 k \gamma \alpha .
\end{aligned}
$$


Note that, the set of eigenvalues $\tilde{\mu}_{j, i}^{(k)}$ repeats $\left(\begin{array}{l}k \\ j\end{array}\right)=\frac{k !}{j !(k-j) !}$ times, for $j=0, \ldots, k$. Also note that, for a fixed $j$, the eigenvalues in the set $\tilde{\mu}_{j, i}^{(k)}$ are all distinct iff all the $\omega_{i}$ are, for $i=0, \ldots, N_{0}-1$.

In the next section, we explore analytically the conditions for the network $\Omega^{(k)}$ to have extensive behaviour. Here, extensivity implies that the sum of the positive LEs, $H_{K S}=$ $\sum_{\lambda_{i}^{(k)}>0} \lambda_{i}^{(k)}$, is a linear function of the size of the system, $N_{k}$.

\section{Results}

As we have seen, the LEs of $\Omega^{(k)}$ are computed by:

$$
\lambda_{i}^{(k)}=\ln \left|2-\mu_{i}^{(k)}\right|
$$

where $\mu_{i}^{(k)}$ are the eigenvalues of $\mathbf{M}_{k}$, for $i=0, \ldots, N_{k}-1$. The LEs of $\Omega^{(k)}$ can be divided into two sets, $\Lambda_{\varepsilon}$ (computed from $\tilde{\mu}_{0, i}^{(k)}$, for $i=0, \ldots, N_{0}-1$ ), that represents the LEs that only depend on the intra-coupling strength, and $\Lambda_{\gamma, \varepsilon}$ (computed from the eigenvalues $\tilde{\mu}_{j, i}^{(k)}$, for $j=1, \ldots, k$ and $\left.i=0, \ldots, N_{0}-1\right)$, formed by the ones that depend on both, the intra and inter-coupling strengths (Baptista et al. 2016; Martín-Hernández et al. 2014). We have explored analytically the conditions for the network $\Omega^{(k)}$ to have the sum of the positive LEs extensive in three different situations.

\section{Case 1}

In our first analysis we consider networks having only positive LEs, which leads to maximal values of $H_{K S}$. Then, assuming that all elements of $\Lambda_{\varepsilon}$ and $\Lambda_{\gamma, \varepsilon}$ are positive we have that:

$$
\begin{aligned}
\ln \left|2-\varepsilon \omega_{i}\right| & >0 \text { and, } \\
\ln \left|2-\varepsilon \omega_{i}-2 j \gamma \alpha\right| & >0
\end{aligned}
$$

for every $i=0, \ldots, N_{0}-1$ and $j=1, \ldots k$. Therefore:

$$
\begin{aligned}
H_{K S} & =\sum_{i=0}^{N_{k}-1} \lambda_{i}=\sum_{i=0}^{N_{k}-1} \ln \left|2-\mu_{i}^{(k)}\right|=\sum_{j=0}^{k} \sum_{i=0}^{N_{0}-1}\left(\begin{array}{l}
k \\
j
\end{array}\right) \ln \left|2-\tilde{\mu}_{j, i}^{(k)}\right| \\
& =\sum_{j=0}^{k} \sum_{i=0}^{N_{0}-1}\left(\begin{array}{l}
k \\
j
\end{array}\right) \ln \left|2-\varepsilon \omega_{i}-2 j \gamma \alpha\right| \\
& =\sum_{j=0}^{k} \sum_{i=0}^{N_{0}-1}\left(\begin{array}{l}
k \\
j
\end{array}\right) \ln \left|2\left(1-\frac{\varepsilon \omega_{i}}{2}-j \gamma \alpha\right)\right| \\
& =\sum_{j=0}^{k} \sum_{i=0}^{N_{0}-1}\left(\begin{array}{l}
k \\
j
\end{array}\right)\left\{\ln (2)+\ln \left|1-\frac{\varepsilon \omega_{i}}{2}-j \gamma \alpha\right|\right\} \\
& =\sum_{j=0}^{k}\left(\begin{array}{l}
k \\
j
\end{array}\right) \sum_{i=0}^{N_{0}-1} \ln (2)+\sum_{j=0}^{k} \sum_{i=0}^{N_{0}-1}\left(\begin{array}{l}
k \\
j
\end{array}\right) \ln \left|1-\frac{\varepsilon \omega_{i}}{2}-j \gamma \alpha\right|
\end{aligned}
$$




$$
\begin{aligned}
& =2^{k} N_{0} \ln (2)+\sum_{j=0}^{k} \sum_{i=0}^{N_{0}-1}\left(\begin{array}{l}
k \\
j
\end{array}\right) \ln \left|1-\frac{\varepsilon \omega_{i}}{2}-j \gamma \alpha\right| \\
& =N_{k} \ln (2)+\sum_{j=0}^{k} \sum_{i=0}^{N_{0}-1}\left(\begin{array}{l}
k \\
j
\end{array}\right) \ln \left|1-\frac{\varepsilon \omega_{i}}{2}-j \gamma \alpha\right| .
\end{aligned}
$$

Equation (33) does not allow to determine explicitly the dependence of $H_{K S}$ on $N_{k}$. To this analysis we will expand the logarithm function. In order to consider the Maclaurin expansion up to first order of $\ln \left|1-\frac{\varepsilon \omega_{i}}{2}-j \gamma \alpha\right|$ in Eq. (33), assume that:

$$
\left|\frac{\varepsilon \omega_{i}}{2}+j \gamma \alpha\right| \ll 1
$$

for every $i=0, \ldots, N_{0}-1$ and $j=0, \ldots, k$. Consequently, for each $i$ and $j$ we have that:

$$
0<1-\frac{\varepsilon \omega_{i}}{2}-j \gamma \alpha \ll 2,
$$

and then, we can omit the absolute value in Eq. (33). Rewriting it we have:

$$
\begin{aligned}
H_{K S} & \simeq N_{k} \ln (2)+\sum_{j=0}^{k} \sum_{i=0}^{N_{0}-1}\left(\begin{array}{l}
k \\
j
\end{array}\right)\left(-\frac{\varepsilon \omega_{i}}{2}-j \gamma \alpha\right) \\
& =N_{k} \ln (2)-\sum_{j=0}^{k}\left(\begin{array}{l}
k \\
j
\end{array}\right) \sum_{i=0}^{N_{0}-1} \frac{\varepsilon \omega_{i}}{2}-\sum_{i=0}^{N_{0}-1} \sum_{j=0}^{k}\left(\begin{array}{l}
k \\
j
\end{array}\right) j \gamma \alpha \\
& =N_{k} \ln (2)-2^{k-1} \varepsilon \sum_{i=0}^{N_{0}-1} \omega_{i}-N_{0} \gamma \alpha \sum_{j=0}^{k}\left(\begin{array}{l}
k \\
j
\end{array}\right) j \\
& =N_{k} \ln (2)-2^{k-1} \varepsilon \sum_{i=0}^{N_{0}-1} \omega_{i}-N_{0} \gamma \alpha\left(k 2^{k-1}\right) \\
& =N_{k} \ln (2)-2^{k-1} \varepsilon S-2^{k-1} N_{0} \alpha \gamma k \\
& =N_{k} \ln (2)-\frac{N_{k}}{2 N_{0}} \varepsilon S-\frac{N_{k}}{2} \alpha \gamma k \\
& =N_{k}\left(\ln (2)-\frac{\varepsilon S}{2 N_{0}}\right)-\frac{N_{k}}{2} \alpha \gamma k
\end{aligned}
$$

where:

$$
S=\sum_{i=0}^{N_{0}-1} \omega_{i}, \quad a=\ln (2)-\frac{\varepsilon S}{2 N_{0}} \quad \text { and } \quad b(k)=-\frac{N_{k}}{2} \alpha \gamma k .
$$

It is now clear the dependence of $H_{K S}$ on $N_{k}$. Let us discuss some of the main features of this. Note that, once we have set the building block with an intra-coupling strength $\varepsilon$, the number $a$ in Eq. (43) is a constant and does not depend on $k$. However, the same is not true for the term $b(k)$, that as we can see, depend strongly on the number $k$. Our goal is to have $H_{K S}$ as a linear function of $N_{k}$, which implies in having $b(k)$ as a constant or only proportional to $N_{k}$. Therefore, in order to satisfy the latter, i.e., $b(k)=d N_{k}$, where $d$ is a constant, we need to require that $\gamma \propto \frac{1}{k}$. Then, choose $\gamma=\gamma(k)=\frac{c}{k}$, for a positive constant $c$. Therefore, 


$$
b(k)=-\frac{\alpha c N_{k}}{2} \text { and } H_{K S} \simeq a^{\prime} N_{k},
$$

where $a^{\prime}=a-\frac{\alpha c}{2}$.

On the other hand, in order to satisfy conditions (25) and (26) without contradicting inequality (34) we must have:

$$
\varepsilon \omega_{i}<1 \text { and } \varepsilon \omega_{i}+2 j \alpha \gamma<1
$$

for each $i=0, \ldots, N_{0}-1$ and $j=1, \ldots, k$. Note that, $0 \leqslant \omega_{i} \leqslant N_{0}$ for each $i=0, \ldots, N_{0}-$ 1 and remember we are considering that $\alpha=1$. Then, aiming to satisfy conditions (34) and (46) simultaneously we need to choose the parameters $\varepsilon$ and $\gamma$ such that:

$$
\begin{aligned}
& \varepsilon \ll \frac{1}{N_{0}} \text { and, } \\
& \gamma \ll \frac{1-\varepsilon N_{0}}{2 k} .
\end{aligned}
$$

Combining condition (48) with the fact that $\gamma$ should be taken inversely proportional to $k$, we have that:

$$
\gamma \equiv \gamma(k)=\frac{1-\varepsilon N_{0}}{2 k C},
$$

for a constant $C$, such that $C \gg 1$. Therefore, if conditions (47) and (49) are satisfied we maintain extensivity keeping all LEs positive as the network grows.

For the networks considered and that have a great deal of symmetry, connectivity and size must be interlinked to create extensivity. In single networks, fully connected, the rescaling to create extensivity would require $\varepsilon$ to be much less than $\frac{1}{N_{0}}$. To networks considered in here, our results show that, the intra-coupling must be bounded by the inverse of the number of nodes of the building block, which can be set as a constant, before the growing process starts. It is the inter-coupling that must be rescaled, and the rescaling depends on the number of times (given by $k$ ) that the network has evolved.

\section{Case 2}

In the second case, we assume that $\Omega^{(k)}$ has positive as well as negative LEs and that the inter-coupling strength $\gamma$ is responsible for the change in the sign of the LEs, i.e., all elements of $\Lambda_{\varepsilon}$ are positive and we do not have any restrictions for the ones that comes from $\Lambda_{\gamma, \varepsilon}$. Then:

$$
\ln \left|2-\varepsilon \omega_{i}\right|>0
$$

for every $i=0, \ldots, N_{0}-1$. Let $n$ be the number of negative LEs and define by $p$ the number of positive ones. For each $i \in\left\{0, \ldots, N_{0}-1\right\}$ and $j \in\{1, \ldots, k\}$ define:

$$
\lambda_{i j}=\ln \left|2-\varepsilon \omega_{i}-2 j \gamma \alpha\right| .
$$

Then, consider the set $\mathcal{A}$ defined by:

$$
\mathcal{A}=\left\{\lambda_{i_{l j l}} \mid i_{l}=i, j_{l}=j \text { and } \lambda_{i j}>0\right\},
$$

i.e., $\mathcal{A}$ is formed by the elements $\lambda_{i j}$ that are positive. Let $q$ be its cardinality. The set $\mathcal{A}$ may contain repeated elements, for instance, if the LE $\lambda_{i j}$ appears " $\times$ " times, then $\lambda_{i j}$ contributes " $\times$ " times in the cardinality of $\mathcal{A}$. 
Therefore, the sum of the positive LEs is given by:

$$
\begin{aligned}
& H_{K S}=\sum_{i=0}^{N_{0}-1} \ln \left|2-\varepsilon \omega_{i}\right|+\sum_{l=1}^{q}\left(\begin{array}{l}
k \\
j_{l}
\end{array}\right) \lambda_{i_{l} j_{l}} \\
& =\sum_{i=0}^{N_{0}-1} \ln \left|2-\varepsilon \omega_{i}\right|+\sum_{l=1}^{q}\left(\begin{array}{l}
k \\
j_{l}
\end{array}\right) \ln \left|2-\varepsilon \omega_{i_{l}}-2 j_{l} \alpha \gamma\right| \\
& =\sum_{i=0}^{N_{0}-1} \ln \left|2\left(1-\frac{\varepsilon \omega_{i}}{2}\right)\right|+\sum_{l=1}^{q}\left(\begin{array}{l}
k \\
j_{l}
\end{array}\right) \ln \left|2\left(1-\frac{\varepsilon \omega_{i_{l}}}{2}-j_{l} \alpha \gamma\right)\right| \\
& =\ln (2)\left(N_{0}+\sum_{l=1}^{q}\left(\begin{array}{l}
k \\
j_{l}
\end{array}\right)\right)+\sum_{i=0}^{N_{0}-1} \ln \left|1-\frac{\varepsilon \omega_{i}}{2}\right| \\
& +\sum_{l=1}^{q}\left(\begin{array}{l}
k \\
j_{l}
\end{array}\right) \ln \left|1-\frac{\varepsilon \omega_{i_{l}}}{2}-j_{l} \alpha \gamma\right| \text {. }
\end{aligned}
$$

As previously, we expand in Maclaurin series up to first order the logarithmic expressions in Eq. (56) to understand the explicit dependence of $H_{K S}$ on $N_{k}$. For that, assume that:

$$
\left|\frac{\varepsilon \omega_{i}}{2}\right| \ll 1 \text { and }\left|\frac{\varepsilon \omega_{i_{l}}}{2}+j_{l} \alpha \gamma\right| \ll 1 \text {, }
$$

for every $i=0, \ldots, N_{0}-1$ and $l=1, \ldots, q$. Note that, $p=N_{0}+\sum_{l=1}^{q}\left(\begin{array}{l}k \\ j_{l}\end{array}\right)$. Then, if $z$ denotes the number of LEs that is equal to zero, Eq. (56) can be rewritten as:

$$
\begin{aligned}
H_{K S} & \simeq p \ln (2)-\sum_{i=0}^{N_{0}-1} \frac{\varepsilon \omega_{i}}{2}-\sum_{l=1}^{q}\left(\begin{array}{l}
k \\
j_{l}
\end{array}\right)\left(\frac{\varepsilon \omega_{i_{l}}}{2}+j_{l} \alpha \gamma\right) \\
& =\left(N_{k}-n-z\right) \ln (2)-\frac{\varepsilon}{2} \sum_{i=0}^{N_{0}-1} \omega_{i}-\frac{\varepsilon}{2} \sum_{l=1}^{q}\left(\begin{array}{l}
k \\
j_{l}
\end{array}\right) \omega_{i_{l}}-\alpha \gamma \sum_{l=1}^{q}\left(\begin{array}{l}
k \\
j_{l}
\end{array}\right) j_{l} \\
& =N_{k} \ln (2)-n \ln (2)-z \ln (2)-\frac{\varepsilon}{2} S-\frac{\varepsilon}{2} \sum_{l=1}^{q}\left(\begin{array}{l}
k \\
j_{l}
\end{array}\right) \omega_{i_{l}}-\alpha \gamma \sum_{l=1}^{q}\left(\begin{array}{l}
k \\
j_{l}
\end{array}\right) j_{l} \\
& =a N_{k}+b(k)+c
\end{aligned}
$$

where:

$$
\begin{gathered}
S=\sum_{i=0}^{N_{0}-1} \omega_{i}, \quad a=\ln (2), \quad c=\frac{-\varepsilon S}{2} \text { and } \\
b(k)=-\left(n \ln (2)+z \ln (2)+\frac{\varepsilon}{2} \sum_{l=1}^{q}\left(\begin{array}{l}
k \\
j_{l}
\end{array}\right) \omega_{i_{l}}+\alpha \gamma \sum_{l=1}^{q}\left(\begin{array}{l}
k \\
j_{l}
\end{array}\right) j_{l}\right) .
\end{gathered}
$$

In this case, the expansion of the logarithm alone was not sufficient to clarify whether $H_{K S}$ is a linear function of $N_{k}$. This is so because of the several terms appearing in 
$b(k)$. But, other terms in this expansion allow us to place some necessary conditions for extensivity. Once we have chosen the building block, the number $c$ is a constant and to have a linear function in Eq. (61), the factor $b(k)$ has to be a constant or a linear function of $N_{k}$.

On the other hand, a solution for the simultaneous inequalities (50) and (57) is:

$$
\begin{gathered}
\varepsilon \ll \frac{1}{N_{0}} \text { and, } \\
\gamma \ll \frac{2-\varepsilon N_{0}}{2 k} .
\end{gathered}
$$

Then, we conclude that, in order to grow the network extensively, keeping positive all the LEs that comes from $\Lambda_{\varepsilon}$, the parameters $\varepsilon$ and $\gamma$ must satisfy conditions (64) and (65) simultaneously and they also should be chosen such that $b(k)$ is a constant or a linear function of $N_{k}$. In the next section, we provide two explicit examples of networks whose parameters satisfy these conditions. We will calculate the terms of $b(k)$, which will strongly depend on the topology of the chosen networks and have to be calculated case by case. This is a contrasts to case 1 , where $b(k)$ does not explicitly depend on the topology.

Another contrast to case 1, in which all LEs are positive, is that the rescaling for the inter-coupling strength is less restrictive and allows a large variation of it. This reflects the fact that, if the building block is set to be chaotic and those blocks are connected in a proper way then this chaotic behaviour propagates throughout the whole network as the network evolves and it is sufficient to induce extensivity, despite that negative LEs appear. This becomes mathematically evident when $H_{K S}$ of the network receives contributions from the positive LEs of the building block even though there exists negative LEs. The inter-coupling is not destroying the local chaotic behaviour of the building blocks. In this sense, chaos also has an extensive behaviour.

\section{Case 3}

In the last case, as in the previous one, we assume that $\Omega^{(k)}$ has positive as well as negative LEs. However, here is only the intra-coupling strength $\varepsilon$ that is responsible for the change in the sign of the LEs, in other words, we are requiring that all elements of $\Lambda_{\gamma, \varepsilon}$ are positive and we do not have any limitations for the ones that comes from $\Lambda_{\varepsilon}$. Then:

$$
\ln \left|2-\varepsilon \omega_{i}-2 j \alpha \gamma\right|>0
$$

for every $i=0, \ldots, N_{0}-1$ and $j=1, \ldots, k$. As before, $n, p$ and $z$ represent the number of negative, positive and null LEs, respectively. Define:

$$
\lambda_{i}=\ln \left|2-\varepsilon \omega_{i}\right| \quad \text { and } \mathcal{B}=\left\{\lambda_{i_{l}} \mid i=i_{l} \text { and } \lambda_{i}>0\right\} .
$$

Let $r$ be the cardinality of $\mathcal{B}$ and, as in the previous case, repeated elements contribute multiple times with $r$. Then, $H_{K S}$ is given by: 


$$
\begin{aligned}
& H_{K S}=\sum_{l=1}^{r} \ln \left|2-\varepsilon \omega_{i_{l}}\right|+\sum_{j=1}^{k} \sum_{i=0}^{N_{0}-1}\left(\begin{array}{l}
k \\
j
\end{array}\right) \ln \left|2-\varepsilon \omega_{i}-2 j \alpha \gamma\right| \\
& =\sum_{l=1}^{r} \ln \left|2\left(1-\frac{\varepsilon \omega_{i_{l}}}{2}\right)\right|+\sum_{j=1}^{k} \sum_{i=0}^{N_{0}-1}\left(\begin{array}{l}
k \\
j
\end{array}\right) \ln \left|2\left(1-\frac{\varepsilon \omega_{i}}{2}-j \alpha \gamma\right)\right| \\
& =\sum_{l=1}^{r} \ln (2)+\sum_{l=1}^{r} \ln \left|1-\frac{\varepsilon \omega_{i_{l}}}{2}\right|+\sum_{j=1}^{k} \sum_{i=0}^{N_{0}-1}\left(\begin{array}{l}
k \\
j
\end{array}\right) \ln (2) \\
& +\sum_{j=1}^{k} \sum_{i=0}^{N_{0}-1}\left(\begin{array}{l}
k \\
j
\end{array}\right) \ln \left|1-\frac{\varepsilon \omega_{i}}{2}-j \alpha \gamma\right| \\
& =\left(r+\left(2^{k}-1\right) N_{0}\right) \ln (2)+\sum_{l=1}^{r} \ln \left|1-\frac{\varepsilon \omega_{i_{l}}}{2}\right| \\
& +\sum_{j=1}^{k} \sum_{i=0}^{N_{0}-1}\left(\begin{array}{l}
k \\
j
\end{array}\right) \ln \left|1-\frac{\varepsilon \omega_{i}}{2}-j \alpha \gamma\right| \\
& =N_{k} \ln (2)-n \ln (2)-z \ln (2)+\sum_{l=1}^{r} \ln \left|1-\frac{\varepsilon \omega_{i_{l}}}{2}\right| \\
& +\sum_{j=1}^{k} \sum_{i=0}^{N_{0}-1}\left(\begin{array}{l}
k \\
j
\end{array}\right) \ln \left|1-\frac{\varepsilon \omega_{i}}{2}-j \alpha \gamma\right| \text {. }
\end{aligned}
$$

since $p=r+\left(2^{k}-1\right) N_{0}=N_{k}-n-z$.

We now expand the logarithm function to analyse the dependence of $H_{K S}$ on $N_{k}$. In order to consider the expansion in Maclaurin series up to first order of the last two terms in Eq. (72) assume that:

$$
\left|\frac{\varepsilon \omega_{i_{l}}}{2}\right| \ll 1 \text { and }\left|\frac{\varepsilon \omega_{i}}{2}+j \alpha \gamma\right| \ll 1 \text {, }
$$

for every $l=1, \ldots, r, i=0, \ldots, N_{0}-1$ and $j=1, \ldots, k$. Therefore, Eq. (72) can be written as:

$$
\begin{aligned}
H_{K S} \simeq & N_{k} \ln (2)-n \ln (2)-z \ln (2)-\sum_{l=1}^{r} \frac{\varepsilon \omega_{i_{l}}}{2}-\sum_{j=1}^{k} \sum_{i=0}^{N_{0}-1}\left(\begin{array}{l}
k \\
j
\end{array}\right) \frac{\varepsilon \omega_{i}}{2} \\
& -\sum_{j=1}^{k} \sum_{i=0}^{N_{0}-1}\left(\begin{array}{l}
k \\
j
\end{array}\right) j \alpha \gamma \\
= & N_{k} \ln (2)-n \ln (2)-z \ln (2)-\frac{\varepsilon}{2} \sum_{l=1}^{r} \omega_{i_{l}}-\frac{\varepsilon}{2}\left(2^{k}-1\right) \sum_{i=0}^{N_{0}-1} \omega_{i} \\
& -N_{0} \alpha \gamma \sum_{j=1}^{k}\left(\begin{array}{l}
k \\
j
\end{array}\right) j \\
= & N_{k} \ln (2)-n \ln (2)-z \ln (2)-\frac{\varepsilon}{2} \sum_{l=1}^{r} \omega_{i_{l}}-\frac{\varepsilon}{2}\left(2^{k}-1\right) S \\
& -N_{0} \alpha \gamma\left(k 2^{k-1}\right)
\end{aligned}
$$




$$
\begin{aligned}
= & N_{k} \ln (2)-n \ln (2)-z \ln (2)-\frac{\varepsilon}{2} \sum_{l=1}^{r} \omega_{i_{l}}-\varepsilon S \frac{N_{k}}{2 N_{0}}+\frac{\varepsilon S}{2} \\
& -\alpha \gamma k \frac{N_{k}}{2} \\
= & N_{k}\left(\ln (2)-\frac{\varepsilon S}{2 N_{0}}\right)+\left(-n \ln (2)-z \ln (2)-\alpha \gamma k \frac{N_{k}}{2}\right) \\
& +\left(\frac{\varepsilon S}{2}-\frac{\varepsilon}{2} \sum_{l=1}^{r} \omega_{i_{l}}\right) \\
& =a N_{k}+b(k)+c,
\end{aligned}
$$

where:

$$
\begin{aligned}
S & =\sum_{i=0}^{N_{0}-1} \omega_{i}, \quad a=\ln (2)-\frac{\varepsilon S}{2 N_{0}}, \quad c=\frac{\varepsilon S}{2}-\frac{\varepsilon}{2} \sum_{l=1}^{r} \omega_{i_{l}} \text { and } \\
b(k) & =-\left(n \ln (2)+z \ln (2)+\alpha \gamma k \frac{N_{k}}{2}\right) .
\end{aligned}
$$

Suppose we have set the building block, then, the number $c$ is a constant and to have the sum of the positive LEs as a function of $N_{k}$ we need to choose the inter-coupling strength such that $\gamma \propto \frac{1}{k}$. Thus, taking $\gamma=\frac{\tilde{c}}{k}$, for a positive constant $\tilde{c}$, we have:

$$
b(k)=-\left(n \ln (2)+z \ln (2)+\frac{\alpha \tilde{c} N_{k}}{2}\right) .
$$

Now, note that, if inequality (66) and the second inequality (73) are satisfied simultaneously for all $i=0, \ldots, N_{0}-1$ and $j=1, \ldots, k$, we conclude that:

$$
\ln \left|2-\varepsilon \omega_{i}\right|>0,
$$

for all $i=0, \ldots, N_{0}-1$ and then, $c=0$ and $b(k)=-\frac{\alpha \tilde{c} N_{k}}{2}$. Therefore, $H_{K S}$ is summarized as:

$$
H_{K S} \simeq a^{\prime} N_{k}
$$

where $a^{\prime}=\left(a-\frac{\alpha \tilde{c}}{2}\right)$.

Notice that, expression (84) coincides to the one in (45). It shows that if all the LEs from $\Lambda_{\gamma, \varepsilon}$ are positive then all the LEs of $\Lambda_{\varepsilon}$ will also be and case 3 comes down to case 1 .

In summary, we have shown that for the dynamics described in Eq. (1), extensivity can always be achieved when an infinitely large multiplex network is constructed by reproducing at all levels the intra and inter structure. To obtain this, we start with any arbitrary network satisfying Eq. (1), a building block, thus, extensivity is obtained if the intra-coupling strength $\varepsilon$ satisfies condition (47) and then, we only rescale the inter coupling-strength $\gamma$ to be inversely proportional to the number of times the network has evolved. The larger this number is, the smaller the inter-coupling strength needs to be.

It is important to notice that the conditions presented here are sufficient but not necessary to have extensivity, which means that may exist extensive networks that do not satisfy any of our restrictions, for example, networks that do not satisfy Eq. (34). Although, our results do not characterize all extensive networks it gives us a recipe to follow when one intends to reach extensivity: choose a building block and create a larger network connecting many of these with appropriate inter-connections. 


\section{Numerical results}

In this section, we present the result of some simulations in order to illustrate and certify our analytical results.

Figure 2 shows the values of $H_{K S}$ (in blue stars) and a linear fitting (in orange straight line) as the initial building blocks with $N_{0}=5$ nodes are evolved, considering two different topologies: (a) ring and (b) star topology. In both situations, $k=[1,2, \ldots, 10]$ and $\varepsilon=2 \cdot 10^{-4}$, which satisfies inequality (47). For $k=1$, we also chose $\gamma(1)$ satisfying condition (49) for $C=10$ and, as we grew the network we rescaled the inter-coupling strength in accordance to condition (49). As can be seen from Fig. 2, choosing the parameters in this way and rescaling the inter-coupling strength during the evolutionary process according to condition (49), the sum of the positive LEs $\left(H_{K S}\right)$ is a linear function of the number of nodes $\left(N_{k}\right)$. This line passes through the origin, which agrees with Eq. (45). In order to measure the error in considering these linear fittings we also calculated the residual standard deviation (Younger 1985), that provides an indication of how close our estimation are. This quantity for both topologies is equal to 0.0036 , which shows that these lines fit very well to the actual data. It also illustrates the fact that, the topology of the building block is not relevant to achieve extensivity. On the other hand, note that, the graphs (a) and (b) are very similar. This is because $H_{K S}$ in each topology only differ from each other due to the factor $S$, as can be seen in Eq. (42) and for these particular topologies this number is very close. For the ring topology we have that $S=10$ and for the star topology $S=8$, what makes the graphs seem almost the same.

Figure 3 shows the values of $H_{K S}$ as a function of $N_{k}$ for networks in case 2. The stars in blue represent the exact values of $H_{K S}$ calculated using Eq. (56). The red star points represent $H_{K S}$ values calculated using the Maclaurin expansion in Eq. (60), whose terms will be rewritten in the following in terms of the network configuration chosen and their relevant parameters. The straight lines are linear fittings. We considered a building block with $N_{0}=5$ nodes and intra-coupling strength $\varepsilon=2 \cdot 10^{-4}$ in two different topologies: (a) ring and (b) all-to-all topology. As in the previous examples, $k=[1,2, \ldots, 10]$, but here for each network $\Omega^{(k)}$ we chose the inter-coupling $\gamma(k)$ satisfying:

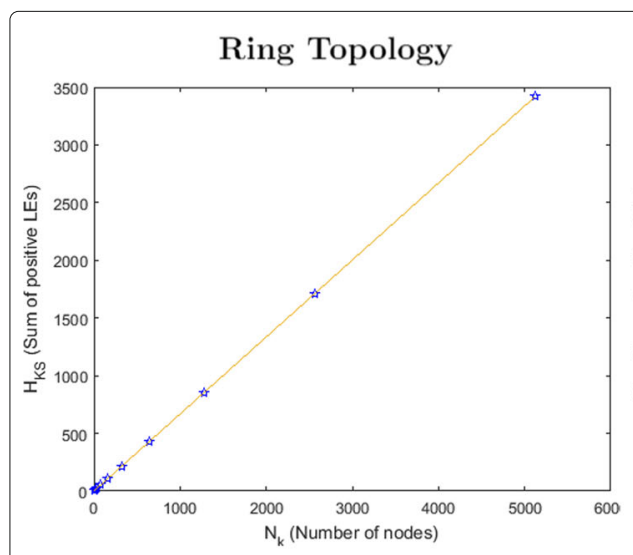

a

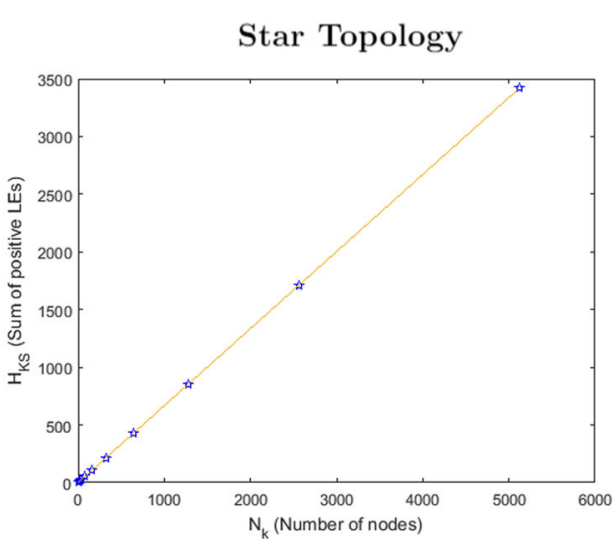

b

Fig. 2 The sum of positive LEs with respect to the number of nodes for networks satisfying case 1 with (a) ring and (b) star topology. For each network we considered the building block with $N_{0}=5$ and $\varepsilon=2 \cdot 10^{-4}$. The inter-coupling of the network $\Omega^{(1)}$ is $\gamma(1)=0.05$ and $k=[1,2, \ldots, 10]$. The fitting in (a) produces $p_{1}=0.6676$ and $p_{2}=-0.0057$ and the one in $(\mathbf{b})$ produces $p_{1}=0.6677$ and $p_{2}=-0.0057$, where $p_{1}$ is the slope and $p_{2}$ is the constant coefficient of the fitting 


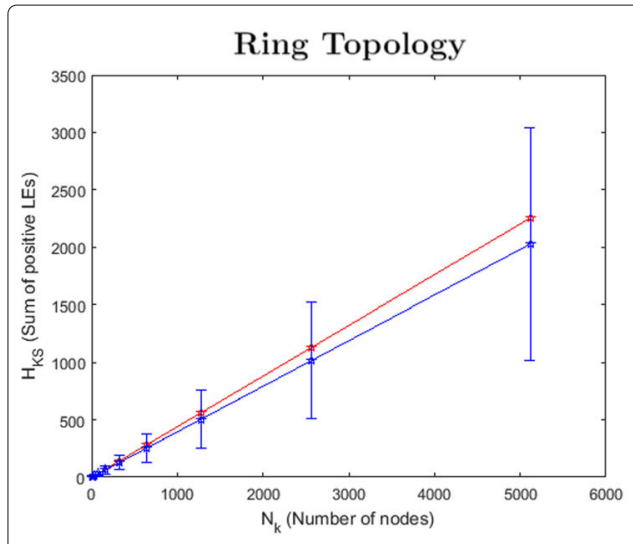

a

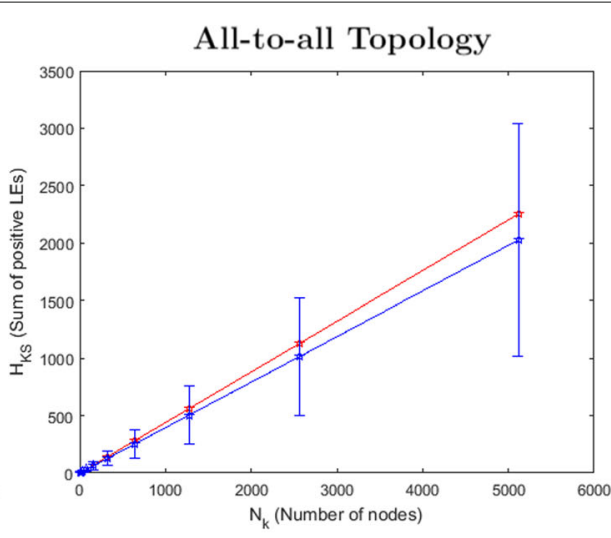

b

Fig. 3 The sum of positive LEs with respect to the number of nodes for networks satisfying case 2 with (a) ring and (b) all-to-all topology. For each network we considered the building block with $N_{0}=5$ and $\varepsilon=2 \cdot 10^{-4}$. The inter-coupling of the network $\Omega^{(1)}$ is $\gamma(1)=0.5045$ and $k=[1,2, \ldots, 10]$. In $(\mathbf{a})$, the linear fitting in the blue line has $p_{1}=0.3965$ and $p_{2}=-0.9896$, and the one in the red line has $p_{1}=0.4407$ and $p_{2}=-0.9422$. $\operatorname{In}(\mathbf{b})$, the linear fitting in the blue line has $p_{1}=0.3962$ and $p_{2}=-0.9881$, and the one in the red line has $p_{1}=0.4405$ and $p_{2}=-0.9412$, where $p_{1}$ is the slope and $p_{2}$ is the constant coefficient of the fitting

$$
\gamma(k)=\frac{\left(1-\varepsilon N_{0}\right)}{2 k}+\frac{1}{2 k C},
$$

for $C=100$. Then, for the network $\Omega^{(1)}$, we have that $\gamma(1)=0.5045$. Note that, the intracoupling strength satisfies condition (64) and choosing $\gamma(k)$ satisfying Eq. (85), we have that, $\gamma(k)$ satisfies condition (65) but does not satisfy constraint (48), i.e., we may have negative LEs.

In order to compute the term $b(k)$ in Eq. (63), for each $k \in\{1, \ldots, 10\}$, we need to estimate the number of negative and null LEs as well as to identify the pairs $(i, j)$ for which $\lambda_{i j}$ in Eq. (51) are positive. For these two examples, our simulations have shown that, for each network $\Omega^{(k)}$, there are no null LEs and the pairs that contribute to the negative LEs are only the pairs $(i, k)$, for $i=0, \ldots, N_{0}-1$, therefore, the number of negative LEs of $\Omega^{(k)}$ is $N_{0}$ and from Eq. (63) we have that:

$$
b(k)=-\left(N_{0} \ln (2)+\frac{\varepsilon}{2} s_{1}(k)+\alpha \gamma(k) s_{2}(k)\right),
$$

where:

$$
\begin{aligned}
\alpha & =1, \\
s_{1}(k) & =\sum_{i=0}^{N_{0}-1} \sum_{j=1}^{k-1}\left(\begin{array}{l}
k \\
j
\end{array}\right) \omega_{i}=S\left(2^{k}-2\right) \quad \text { and }, \\
s_{2}(k) & =N_{0} \sum_{j=1}^{k-1}\left(\begin{array}{l}
k \\
j
\end{array}\right) j=N_{0} k\left(2^{k-1}-1\right) .
\end{aligned}
$$

Consequently, Eq. (86) becomes:

$$
b(k)=-5 \ln (2)-\varepsilon S 2^{k-1}+\varepsilon S-\gamma(k) N_{0} k 2^{k-1}+\gamma(k) N_{0} k .
$$

Rewriting Eq. (85) as:

$$
\gamma(k)=\frac{C^{\prime}}{k}, \quad \text { where: } \quad C^{\prime}=\frac{\left(1-\varepsilon N_{0}\right) C+1}{2 C},
$$


we have that:

$$
\begin{aligned}
b(k) & =-5 \ln (2)-\varepsilon S 2^{k-1}+\varepsilon S-C^{\prime} N_{0} 2^{k-1}+C^{\prime} N_{0} \\
& =-5 \ln (2)-\varepsilon S \frac{N_{k}}{2 N_{0}}+\varepsilon S-C^{\prime} \frac{N_{k}}{2}+C^{\prime} N_{0} \\
& =N_{k}\left(-\frac{\varepsilon S}{2 N_{0}}-\frac{C^{\prime}}{2}\right)+\left(-5 \ln (2)+\varepsilon S+C^{\prime} N_{0}\right) .
\end{aligned}
$$

Then, from Eq. (61) the approximate sum of the positive LEs for each $\Omega^{(k)}$ is given by:

$$
H_{K S} \simeq\left(\ln (2)-\frac{\varepsilon S}{2 N_{0}}-\frac{C^{\prime}}{2}\right) N_{k}+\left(\frac{\varepsilon S}{2}-5 \ln (2)+C^{\prime} N_{0}\right),
$$

that is a linear function of $N_{k}$. Extensivity is achieved as it is confirmed by the blue stars (calculated by Eq. (56)) and the red stars (calculated by the expansion in Eq. (60)) in Fig. 3. Note that, again $H_{K S}$ in both topologies only differ from each other by the factor $\frac{\varepsilon S}{2}$ and, although the difference between the values of $S$ in Fig. 3 is bigger in comparison with the Fig. 2 ( $S=10$ in (a) and $S=20$ in (b)), both plots show similar behaviour. The reason for this is due to $S$ being multiplied by $\varepsilon$ what makes the product $\frac{\varepsilon S}{2}$ very small.

The linear approximations provide a good fitting for the data. For the ring topology, the residual standard deviations are equal to 0.6453 and $9.7 \cdot 10^{-14}$ for the blue and red lines, respectively and, for the all-to-all topology, these quantities are equal to 0.6457 and $1.09 \cdot 10^{-13}$, respectively.

The difference between the blue and red linear fitting lines represents the error of the Maclaurin expansion. To show that this error is an artifact due to the expansion (and therefore, it tends to zero, as the argument of the logarithm approaches 1) and not due to any other analytical miscalculation, we have plot the error bar in blue. This error bar represents the maximal error one could obtain from the approximate values of $H_{K S}$ through the Maclaurin expansion if all the Lyapunov exponents would contribute equally to the total error with the same amount of the error produced by the exponent whose argument inside the logarithm function deviates the most from 1.

Figure $4 \mathrm{a}$ and $\mathrm{b}$ show the exact values for $H_{K S}$ (in blue stars) calculated using Eqs. (33) and (56), respectively, when the conditions specified for cases 1 and 2 to maintain extensivity are not satisfied. In both networks we considered the star topology for the building blocks with $N_{0}=5$ nodes. For each network, we considered $k$ from 1 up to 6 and $\varepsilon=2 \cdot 10^{-4}$, that obeys conditions (47) and (64). With regards to the inter-coupling strength, for the network $\Omega^{(1)}$ we chose $\gamma(1)=0.05$ in (a) and $\gamma(1)=0.5045$ in (b), that satisfy Eq. (49) and condition (65), respectively. In order to show the importance of rescaling the inter-coupling strength as we evolve the network, we kept constant these parameters during the evolutionary process and, as we can see from Fig. 4, $H_{K S}$ does not dependent linearly on the number of nodes of the network. This fact is pretty clear in (b) and in (a) we considered a linear fitting (in the orange straight line) to help this visualization. The residual standard deviation in this case is equal to 1.96, that is much bigger when compared with the one in Fig. 2, showing that the linear regression there is more consistent than here.

Finally, in Fig. 5, graphs (a) and (b) present two additional scenarios for which the conditions to maintain extensivity put forwarded in cases 1 and 2 are not satisfied, respectively. In both situations, we considered the star topology for $\Omega^{(0)}$ with $N_{0}=5$ nodes and we also kept constant all the intra and inter-coupling strengths as in the previous example. 


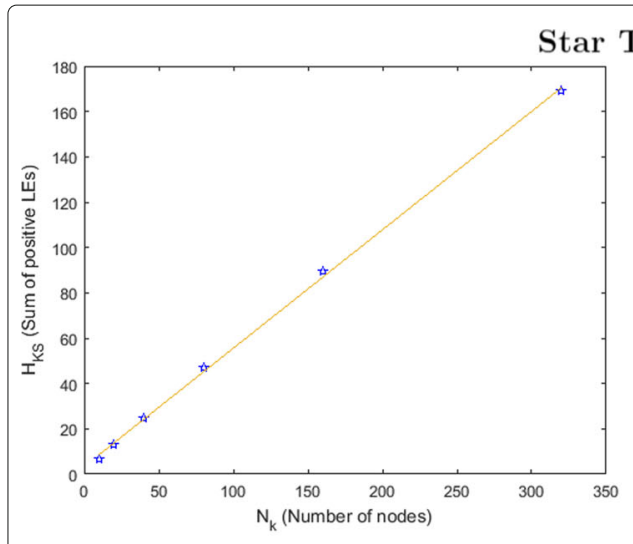

a

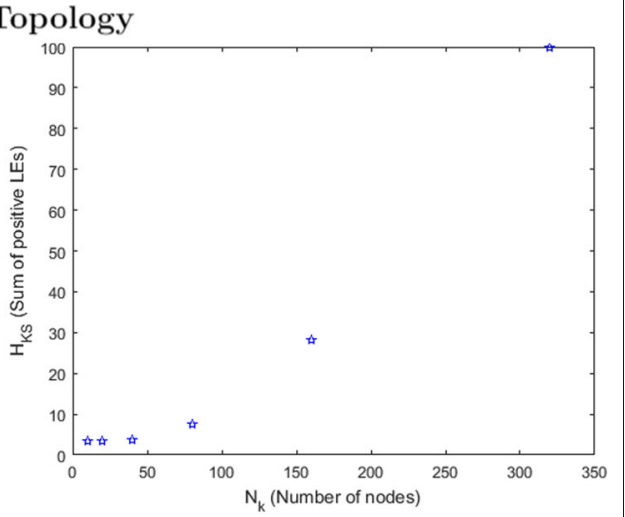

b

Fig. 4 The sum of positive LEs with respect to the number of nodes for networks with star topology that do not satisfy cases 1 and 2, respectively. For each network we considered the building block with $N_{0}=5$ and $\varepsilon=2 \cdot 10^{-4}$. In (a) the inter-coupling of the network $\Omega^{(1)}$ is $\gamma(1)=0.05$ and in (b) $\gamma(1)=0.5045$ and $k=[1,2, \ldots, 6]$. In both graphs, during the evolutionary process the inter-coupling strength was kept constant. The fitting in the orange line in $(\mathbf{a})$ produces $p_{1}=0.5223$ and $p_{2}=3.3490$, where $p_{1}$ is the slope and $p_{2}$ is the constant coefficient of the fitting

However, here we have chosen $\varepsilon=0.19$ that does not obey conditions in (47) or (64). Besides, in scenario (a) we set $\gamma(1)=0.2$, contradicting condition (48) and in scenario (b) $\gamma(1)=0.6$, which also does not satisfy condition (65). As in Fig. 4, we evolved the network 6 times. Figure 5 shows that $H_{K S}$ is not extensive for these chosen parameters. It is worth commenting that whereas in (a) extensivity is not achieved because $H_{K S}$ is a convex shape, in (b) it has a concave shape. This concave shape was argumented in (Antonopoulos and Baptista 2017) to be a finite effect. This kind of behaviour was called super-extensive, when $H_{K S}$ grows faster than any possible linear approximation. Our numerical results do not contradict this logic, however, it is interesting to see that even networks with hundred of nodes can apparently behave as being super-extensive. Finally, these results evidence that the choice of $\varepsilon$ and the inter coupling strength $\gamma(1)$ of $\Omega^{(1)}$ play a fundamental role in maintaining the extensive behaviour of $H_{K S}$ in large multiplex networks.

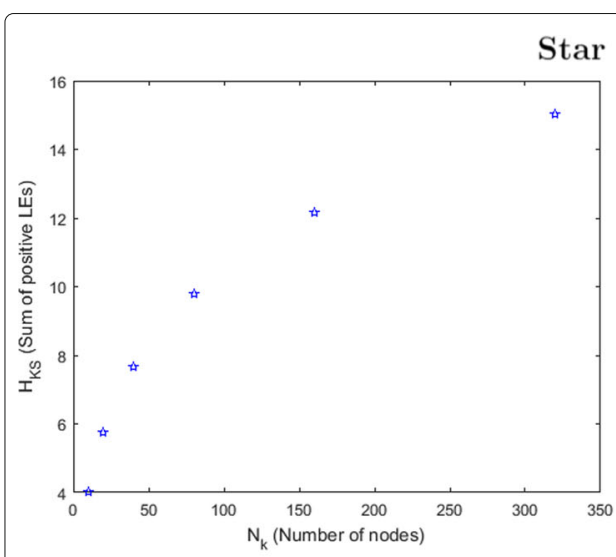

a

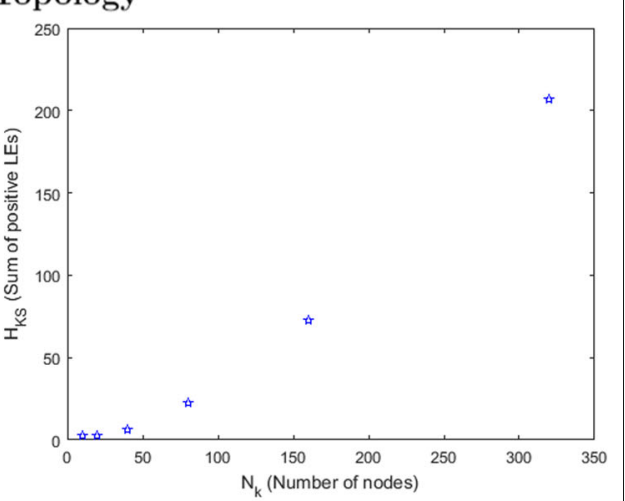

b

Fig. 5 The sum of positive LEs with respect to the number of nodes for networks with star topology that do not satisfy cases 1 and 2, respectively. For each network we considered the building block with $N_{0}=5$ and $\varepsilon=0.19$. In (a) the inter-coupling of the network $\Omega^{(1)}$ is $\gamma(1)=0.2$ and in $(\mathbf{b}) \gamma(1)=0.6$ and $k=[1,2, \ldots, 6]$. In both graphs, during the evolutionary process the inter-coupling strength was kept constant 
In addition to the examples presented in this section we also have checked that our findings hold in networks used to described real-life phenomena and with less trivial topologies such as, the circulant network, the random model of network $G(N, M)$ proposed by Erdös and Rényi, the small-world network using the Watts-Strogatz model and the scale-free network presented by Barbarasi-Albert.

\section{Conclusions}

Multiplex networks are fundamentally different when compared to single layer networks (Gomez et al. 2013) and their sophisticated nature can provide a more realistic picture to model large real-world systems, since most of real life phenomena are described by multiple types of connections. This paper explores the role of the intra and inter-connections to maintain the extensive behaviour of the sum of the positive LEs in infinitely large multiplex networks.

Our analytical results have showed that for the type of networks considered here, it is always possible to construct extensive networks by rescaling only the inter-couplings and keeping the intra-connections constant during the evolutionary process. Due to this growth method, the rescale $\varepsilon=\frac{1}{N}$ (where $N$ stands for the system size), which is broadly adopted by the nonlinear community of academics to study collective behavior of networks of different sizes, is an over underestimation to achieve extensivity. The obtained rescale for the intra-coupling strengths does not depend directly on the size $N$ of the network but on the size $N_{0}$ of the initial building block considered in the process. So, our extensive networks can be constructed with much less restrict rescaling than those used for single layer networks. Consequently, the number of nodes of the initial networks have a major contribution to the extensive behaviour rather than the way the nodes in the initial networks are connected by internally (intra-connection). These results are at variance with the outcomes from the work developed by Antonopoulos and Baptista (2017), where they showed that extensivity depend on other quantities apart from the type of couplings, such as the sum of the intra-degrees and any single network whose the sum of the intradegrees is proportional to $N^{2}$ would be extensive by the $\varepsilon=\frac{1}{N}$ rescaling. In here, a very relevant physical quantity leading to appropriate rescaling is the number of times the network has evolved.

Introducing the term super entropic for networks that present only positive Lyapunov exponents, our findings also reveal that networks that are not super entropic, are easier to be made extensive, since the parameters range to achieve extensivity are larger.

Our main contribution in this work was not only to show which quantities are related to extensivity, but also provide a reliable set of conditions to be obeyed to achieve networks that grows in size still maintaining the extensive character. With regards to the generality of our results, a task left for future work would be to explore whether our main conclusions can be exported to multiplex networks with more complex topologies (by varying $\alpha$ ) or constructed with nodes having more generic dynamical descriptions, such as neural networks.

Abbreviations

LEs: Lyapunov exponents 


\section{Authors' contributions}

This article is a product of MAA's PhD supervisioned by MSB. MAA developed the theoretical formalism, the analytical calculations, performed the numerical simulations and wrote the manuscript. MSB proposed the study and contributed towards the execution and the writing of this work. Both authors read and approved the final manuscript.

\section{Funding}

MAA acknowledges the University of Aberdeen for her Elphinstone PhD scholarship.

\section{Availability of data and materials}

The data and code that support the findings of this study are available from the corresponding author on reasonable request.

\section{Competing interests}

The authors declare that they have no competing interests.

Received: 2 May 2019 Accepted: 23 July 2019

Published online: 23 September 2019

\section{References}

Antonopoulos CG, Baptista MS (2017) Maintaining extensivity in evolutionary multiplex networks. PloS ONE 12(4):e0175389

Baptista MS, Szmoski R, Pereira R, de Souza Pinto SE (2016) Chaotic, informational and synchronous behaviour of multiplex networks. Sci Rep 6:22617

Boccaletti S, Latora V, Moreno Y, Chavez M, Hwang DU (2006) Complex networks: Structure and dynamics. Phys Rep 424(4-5):175-308

Bullmore E, Sporns O (2009) Complex brain networks: graph theoretical analysis of structural and functional systems. Nat Rev Neurosci 10(3):186

Eckmann JP, Ruelle D (1985) Ergodic theory of chaos and strange attractors. In: The Theory of Chaotic Attractors. Springer, New York. pp 273-312

E J Newman M. (2010) Networks: An Introduction. Oxford university press, Oxford

Estrada E (2015) Introduction to complex networks: structure and dynamics. In: Evolutionary Equations with Applications in Natural Sciences. Springer, Switzerland. pp 93-131

Gomez S, Diaz-Guilera A, Gomez-Gardenes J, Perez-Vicente CJ, Moreno Y, Arenas A (2013) Diffusion dynamics on multiplex networks. Phys Rev Lett 110(2):028701

Karimi A, Paul MR (2010) Extensive chaos in the Lorenz-96 model. Chaos: Interdiscip J Nonlinear Sci 20(4):043105

Luccioli S, Olmi S, Politi A, Torcini A (2012) Collective dynamics in sparse networks. Phys Rev Lett 109(13):138103

Martín-Hernández J, Wang H, Van Mieghem P, D'Agostino G (2014) Algebraic connectivity of interdependent networks. Phys A Stat Mech Appl 404:92-105

Monteforte M, Wolf F (2010) Dynamical entropy production in spiking neuron networks in the balanced state. Phys Rev Lett 105(26):268104

Nardelli PH, Rubido N, Wang C, Baptista MS, Pomalaza-Raez C, Cardieri P, et al. (2014) Models for the modern power grid. Eur Phys J Spec Top 223(12):2423-2437

Ott E (2002) Chaos in dynamical systems. 2nd ed.. Cambridge university press, Cambridge

Paul M, Einarsson M, Fischer P, Cross M (2007) Extensive chaos in Rayleigh-Bénard convection. Phys Rev E 75(4):045203

Robins G, Pattison P, Kalish Y, Lusher D (2007) An introduction to exponential random graph ( $p^{*}$ ) models for social networks. Soc Networks 29(2):173-191

Ruelle D (1982) Large volume limit of the distribution of characteristic exponents in turbulence. Commun Math Phys 87(2):287-302

Shibata T, Kaneko K (1998) Collective chaos. Phys Rev Lett 81(19):4116

Stahlke D, Wackerbauer R (2009) Transient spatiotemporal chaos is extensive in three reaction-diffusion networks. Phys Rev E 80(5):056211

Stahlke, D, Wackerbauer R (2011) Length scale of interaction in spatiotemporal chaos. Phys Rev E 83(4):046204

Strogatz SH (2001) Exploring complex networks. Nature 410(6825):268

Takeuchi KA, Chaté H, Ginelli F, Politi A, Torcini A (2011) Extensive and subextensive chaos in globally coupled dynamical systems. Phys Rev Lett 107(12):124101

Takeuchi KA, Ginelli F, Chaté H (2009) Lyapunov analysis captures the collective dynamics of large chaotic systems. Phys Rev Lett 103(15):154103

Xi Hw, Toral R, Gunton JD, Tribelsky MI (2000) Extensive chaos in the Nikolaevskii model. Phys Rev E 62(1):R17

Younger MS (1985) A first course in linear regression. PWS Publishing Co., Boston

\section{Publisher's Note}

Springer Nature remains neutral with regard to jurisdictional claims in published maps and institutional affiliations. 